\title{
THE DEVELOPMENT AND STRUCTURE OF AN ACTIVE REGION*
}

\author{
C. DE JAGER \\ (University Observatory and Space Research Laboratory, Utrecht, The Netherlands)
}

\section{Introduction}

The concept active region, previously called 'centre of activity', was introduced by D'Azambuja as "the totality of all visible phenomena accompanying the birth of sunspots". This idea was first placed in the centre of attention during the Convegno Volta in 1952 when Kiepenheuer put forward his paper: 'Was ist ein Aktivitätszentrum auf der Sonne?'. At that time our knowledge of an active region could still be condensed in a five-page paper. During the present symposium we have listened to 89 communications, all dealing with certain aspects of the active region. Particular attention was given to the role played by supergranulation and by detailed magnetic fields; this was possible since we now have precise observations with good spatial and time resolution of the magnetic field, and very detailed information on the related optical structures.

The present review is called 'Development and Structure of an Active Region', as it is the development that is essential; structures will be dealt with only in as far as they are important for our understanding of the way an active region develops. The mechanism behind the development of an active region may now be defined, with Kiepenheuer, as "the interplay of solar convection, differential rotation, and magnetic fields on the Sun".

A general remark that should be made after this Symposium refers to the difference between the resolution attained optically on one side, and in X-ray and radio observations on the other hand. With optical methods a spatial resolution of the order of $1^{\prime \prime}$ can occasionally be reached; sometimes even better observations are made. Radio and $\mathrm{X}$-ray observations are still in the range of tens of seconds of arc or even minutes of arc. This hinders the intercomparison of coronal observations (which rely on radio and X-ray observations) with photospheric and chromospheric data. One of the most important and imperative developments in radio and $X$-ray astronomy will be to improve the resolution to something around $1^{\prime \prime}$.

* Invited summarizing review of the Budapest Symposium.

Kiepenheuer (ed.), Structure and Development of Solar Active Regions, 602-608. (C) I.A.U. 


\section{Large-Scale Development of the Active Region}

As shown in Kiepenheuer's introductory paper the broad development of an active region can best be understood on the basis of Babcock's theory, but certain problems remain. The first is how to neutralise the poleward moving fields: it appears that the magnetic flux that moves poleward is about ten times larger than the flux eventually arriving. An answer to this problem has been given during the Budapest Symposium by Stenflo: when the weaker fields in and around an active region are taken into account there appears to be a better flux balance in the two magnetically oppositely polarized parts of active regions, than has been assumed hitherto. Beckers and Schröter found that the fields of the magnetic knots partly or wholly balance those of the spots.

A harder problem, not compatible with Babcock's theory, is that apparently new active regions or even new-cycle regions may appear to originate in old ones, or they may occur at the places of old ones. It remains to be examined whether this observation would break down Babcock's theory or not.

A further general aspect concerns the origin of solar active regions. There are clear indications that certain longitudes are favoured. They seem to be situated about $180^{\circ}$ apart; both Mrs. Dodson-Prince and Švestka produced data pointing in that direction. However, as can be seen from a comparison of Figure 1 of Mrs. Dodson-Prince with figure 3 of Švestka, it is not clear whether the two favourite longitudes rotate with the normal photospheric speed (Carrington) or whether they find their basis in subphotospheric regions rotating at a much faster rate.

In any case it seems that the notion plage family may be justified and may merit further attention.

\section{The First Phase of Development of an Active Region}

There are indications that the basic difference between the active and the quiet parts of the Sun is a quantitative rather than a qualitative one. The basic magnetic structures, also visible in the quiet Sun, appear in larger quantities in the active region. We should particularly stress the importance of the supergranulation for the development of solar activity.

In the quiet Sun some essential scales of length may be defined (see also the paper by Simon and Weiss), as given in Table 1.

The magnetic field is concentrated in the supergranulation boundaries where it is directed radially; there is no component at right angles.

Next we describe the various phases of development of an active region. The first phase is the arrival of magnetic flux at the surface. This is a rapid event, occurring during about 1 day; it soon leads to the development of a bipolar magnetic region. Here the question may be asked whether real bipolar magnetic regions do exist or 


\begin{tabular}{lcll}
\multicolumn{1}{c}{ object } & life-time & $\begin{array}{l}\text { Table 1 } \\
\text { characteristic size }\end{array}$ & \multicolumn{1}{c}{ size related to } \\
granulation; spicules & $10 \mathrm{~min}$ & $1000 \mathrm{~km}$ & $\begin{array}{l}\text { scale height in sub-photospheric } \\
\text { region }\end{array}$ \\
$\begin{array}{l}\text { mottles } \\
\text { rosettes } \\
\text { supergranulation }\end{array}$ & $\begin{array}{l}1 \text { day } \\
\text { some days }\end{array}$ & $\begin{array}{l}1000 \mathrm{~km} \\
5000-10000 \mathrm{~km}\end{array}$ & $? \begin{array}{l}\text { scale height somewhere deep in } \\
\text { the convective region }\end{array}$ \\
large network & $?$ & $30000 \mathrm{~km}$ & $\begin{array}{l}\text { giant cells in the deep convective } \\
\text { zone }\end{array}$
\end{tabular}

whether refined magnetic measurements will eventually show that only complex magnetic regions occur on the disk.

Prior to the outbreak of spots the brightness difference in photospheric levels increases in the sense that the intergranular space becomes darker. Slightly later, pores originate; a fine example, from a movie-picture, was given by Rösch. The intensity in the pore is about 0.6 times the continuum intensity. It is very important to realise that the pores and spots never originate inside the supergranulation, but always at the boundaries, apparently due to a concentration of the magnetic flux by the supergranular motion field.

Still obscure is the part played in this connection by the objects called 'magnetic knots', 'invisible spots', or 'satellite spots'. These three terms refer to the same object! The first seems to be the best, since upon more detailed examination the 'invisible' spots nearly always appear to become visible, though they are less dark than the pores; the intensity depression is about $0 \cdot 1$ times the continuum intensity. They have magnetic fields of the order of 1000 gauss (up to 1400 gauss) and diameters between $1^{\prime \prime}$ and 12 ". An important and still open question: what is the role of the magnetic knots in the formation of spots?

During the development of spotgroups, individual spots may show strong motions with respect to each other. After the formation of spots the facular region extends in area: the $\mathrm{K}$ emission appears to spread over the whole supergranular region. At that time the first phase of development of an active region may be considered as ended; the main phase of development sets in; it is characterized by intense chromospheric and coronal activity and in particular by flares. We will discuss the main phase of development of the photospheric and chromospheric parts of the active region in Section 4, the coronal and interplanetary parts in Section 5; flares will be discussed in Section 6.

\section{The Main Phase of Development; (a) Photosphere and Chromosphere}

The areal development of the photospheric and chromospheric facular regions was investigated particularly well during the CSSAR period (Cooperative Study of Solar 
Active Region). It appears that the plages reach their maturity in less than 1 month and decline slowly in activity in a period of the order of 3-5 months. It is known, of course, that occasionally plages may live much longer.

The relation of the facular fine structure and network to the supergranulation and the magnetic field should be mentioned. Plages, in particular those that are not yet too bright, show in chromospheric images a kind of network structure which seems to extend into the quiet chromospheric network. However, it should be made clear that the identity of the network in active regions and a possible active-region supergranulation has not yet been proved. The concept supergranulation is defined by the photospheric-chromospheric motion field; supergranulation is a large structure in which material is moving towards the borders where the material apparently moves downward. At the borderline magnetic fields occur. In the quiet Sun the supergranulation appears to be identical with the chromospheric network; the same need not apply to the network in the active region.

Oscillatory motions have been discovered both in the quiet and in the active regions. Their periods, being of the order of $5 \mathrm{~min}$ in the undisturbed photosphere increases to $45 \mathrm{~min}$ in the disturbed region; the amplitude seems to decrease with increasing field. These motions are correlated to brightness variations; not to variations of the magnetic field.

An important problem with regard to the sunspot is that of the energy balance in spots. This problem, in turn, is directly connected to that of the 'missing energy flux': where does the sub-photospheric convective energy flux go, that does not escape in the form of radiation in the region of a sunspot? This energy flux is large and, if used $100 \%$ efficiently, would provide the energy for a large flare in about $1000 \mathrm{sec}$. Danielson argued that part of this energy flux may escape sidewards in sub-photospheric regions in the form of gravity waves. It is still unclear what fraction of the energy moves up vertically in the form of hydromagnetic waves.

The diameter of the umbral granules may be at most $500 \mathrm{~km}$; but they may even be as small as $160 \mathrm{~km}$. In the latter case the umbral granules would have the same surface brightness as the normal photosphere, which is not impossible; they have the photospheric colour temperature too.

An interesting feature in the chromosphere above active regions is formed by the 'bright points', seen in the $\mathrm{H} \alpha$ wings (moustaches, bombs). They have diameters of $1^{\prime \prime}$ to $5^{\prime \prime}$ and lifetimes of the order of $25 \mathrm{~min}$. Bruzek drew attention to the close relation between these features and his newly discovered arch-shaped filaments. These filaments occur between small areas of opposite polarity in active regions. They cross the neutral line and nearly always connect bright points situated at their bases. Material motions with velocities of the order of some tens of $\mathrm{km}$ per second occur along the filaments which tend to expand.

There is a clear difference between the above-mentioned arched filaments and the other filamentary structures in or above the solar chromosphere: the fibrilles and the 
classical filaments, which are nothing but the prominences projected on the disk. High-resolution pictures show a relation between filaments and the fibrilles: the first may be the result of some shearing motion. It is not unlikely that these fibrilles are related to a sheared magnetic-field structure carrying the filaments. It may be significant that fibrilles situated beyond the ends of filaments are directed more or less perpendicularly to the filamentary direction, whereas the fibrilles at the sides of the filament tend to be inclined at only small angles to it.

\section{Main Phase of Development of an Active Region; (b) the Coronal and Interplanetary Part}

By means of observations of forbidden lines in the visible spectral region the solar corona is observed at the limb; disk observations are made by means of X-ray and radio measurements. In all cases the resolution is bad: at the limb because we integrate over a long line of sight, and on the disk because the observing techniques are still rather primitive.

It appears that the ratio between the intensities of the red and the green coronal lines vary systematically during the development of an active region. A similar behaviour has been found for various ion lines in the EUV. It appears very hard to explain this variation without the introduction of inhomogeneities in the coronal extention of the active region; in particular this seems to be necessary in the beginning phase of the development of the coronal active region; the inhomogeneities may disappear later.

Attempts have been made to compute the coronal magnetic fields from the given photospheric boundary values, assuming a potential field. The results of these computations appear to be in conflict with other observations. This clearly indicates that the coronal parts of active regions are not current-free, at least not at the 'small' scale of the corona. However, it may be that on a larger, interplanetary scale the field is indeed current-free.

It is also clear that the structures above the coronal active regions are for the greater part defined by the magnetic field, which, in turn, is defined by three different quantities: the photospheric field, coronal electrical currents, and the solar wind. It is a general rule that above all active chromospheric regions dense coronal regions occur. The temperature of these dense regions is of the order of $2.5 \times 10^{6}{ }^{\circ} \mathrm{K}$. Recent measurements of the electron densities in coronal active regions show these to be about 2-5 times the quiet coronal density. However, the shape of the active region depends on the magnetic structure and its state of evolution. The well-known coronal bushes and helmets may correspond to closed field lines and occur in the early phase of the life history of the coronal active regions. In later phases one observes the streamers, characterized by their pinching tendency, and the rays, with their sharp structures extending radially from the Sun. The rays have been seen with rocket-borne corona- 
meters, and appear to stretch out to distances of many solar radii. They do not show any pinching tendency and apparently correspond to a structure with completely open field lines.

In the interplanetary medium the sector structure, discovered by Wilcox and Ness, is related to plages in the preceding parts of each of the sectors. It is not impossible that these structures are current-free and correspond to closed field lines.

\section{Flares}

The Proton Flare Project (PFP) organized by Švestka and Simon has appeared to be extremely useful for increasing our knowledge about the very energetic events related to solar flares. Where and when do flares occur?

New additional information has been advanced for showing that flares occur preferably in magnetically complex active regions; in particular near regions where the gradient of the longitudinal field is strong. Flares tend to favour regions where new plages or spotgroups are born, in particular where they are included in old ones. Dr. S. Smith and Howard have mentioned the importance of the reversed polarity regions, which produce more flares than simple bipolar magnetic regions. In particular islands of opposite polarity in large magnetic regions are places favoured by flares. The Ellerman bombs seem to favour the regions where $|B|=0$.

Flares seem to occur in the above-mentioned regions at moments when the magnetic energy of the active region has greatly increased. Some cases have been mentioned by Severny. In one case it appeared that the magnetic energy already started to decrease, a few hours before the occurrence of the flare.

After the occurrence of the flare the transverse field component may appear to have changed its direction by $90^{\circ}$ and tends to be oriented more parallel to the 'neutral' line.

Flares are known to show a repetitive tendency; for radio flares it has been shown that the period during which homologous flares can occur in one active region is not longer than about 1-2 days.

It seems a fairly general rule for plages to show a small decrease in brightness just prior to the brightness increase of the flare; later the integrated $\mathrm{H} \alpha$ brightness may show pulsations.

Optical flares are often accompanied by surges and similar phenomena, indicating the acceleration of a low-temperature plasma. These accelerations seem to be related to the propagation of the fast hydromagnetic mode.

The optical flare plasma finds its counterpart in the high-energy flare plasma. It is a plasma, containing particles accelerated to energies of the order of 10 to a few hundreds of keV's. The lower the average energy of the plasma the larger the amount of accelerated electrons; very roughly the product of the average particle energy with the total number of accelerated electrons is constant. The acceleration to these 
energies takes place in the very first phase of the flare process as shown by X-ray and microwave type-IV radio emission, as well as by type-III radiobursts. A second acceleration, whereby the energy is multiplied by another factor, of the order of $10^{3}$, seems to occur in a later phase of the flare; the metric type-IV emission suggests that it occurs perhaps about a quarter to a full hour later.

Since this later acceleration may be due to a kind of Fermi process, flare theories basically need only explain the first acceleration process. Three different sources for the flare energy have been mentioned. The first is the shearing energy of photospheric motions, a process advocated by Sturrock. Another source may be the 'missing' convective energy flux from below the sunspots which would produce sufficient energy for a large flare in about $15 \mathrm{~min}$ (De Jager). A third possible energy source was mentioned by Danielson and is the turbulent energy flux inside sunspots. However, it is not sufficient simply to mention the energy source; further unknown factors are (a) how to store the energy: perhaps in the form of magnetic energy; and (b) how to free it. As a possible triggering mechanism a current discharge as propagated in studies by Severny and Alfvén might be mentioned.

Little has been said about coronal events occurring in connection with or after flares; radio and direct optical measurements indicate that the electron density in the low corona seems to increase after a flare.

\section{The Last Phases of the Active Region}

With regard to the last phases we only want to make brief mention of a few problems; no clear solutions have as yet been given.

What is the character and the scope of the poleward motion of filaments and the coronal activity; how does it relate to the problem of the dissolution and the partial neutralisation of the magnetic field? What happens to the field lines in the low solar corona in the last phases of the active region; are they neutralised and how does this happen? What is the significance of the ghost fields in relation to the evolution of active regions? Future research may answer these questions. 